\title{
Design and Implementation of Radar Signal Generator
}

\author{
Tarık Ünler ${ }^{*}$ and Levent Seyfi ${ }^{2}$
}

\begin{abstract}
In this study, to design a signal generator which is required for a typical radar is aimed. The frequency of this genarator can be varied rapidly via SPI (Serial Perihpheral Interface). Signal generation is carried out using ADF4350BCPZ chip. UNAOHM spectrum analyzer and KC901S network analyzer are used to make measurements after implemantation of the generator. As a result of this study, RF signals are generated and displayed on spectrum at 7 different frequencies.
\end{abstract}

Index Terms - Signal generator, Radar, PLL, RF.

\section{INTRODUCTION}

Radars are widely used in areas such as defense, civil aviation, meteorology, and speed control applications. Radars, which are indispensable part of defense system, still continue to be developed. Radars produced in many frequency bands also have many types. The base of the radar is signal generator. The generated signal varies depending on the type and structure of the radar to be used. Radar signal is generated in different frequency bands for factors such as high resolution, distance to target, type of target, weather conditions. In addition, it is important for the design to be manufactured that whether the signal to be produced will be continuous or in pulses. Apart from these, power of the signal to be generated is a significant factor to determine the radar range.

Along with improvements in technology, small size, low energy consumption RF element production has become widespread [1]. RF signals can be produced in different ways from conventional methods. One of these methods is the generation of the RF signal as a direct digital synthesis (DDS) with a high-speed FPGA [2]. The signal generated by the DDS is produced by digital switching, unlike the VCO structure. The shape of the signal (sine, triangle, square) is determined by the algorithm within the processor or controller. Another method is to use the transistor element as an oscillator to generate a signal at the desired frequency range with the necessary matching circuits [3]. Because such pulse generators are difficult to produce and are influenced by external components, there are broadband pulse generators produced in CMOS integrated structures too [4], [5].

Because these structures are integrated, they are less affected

\footnotetext{
${ }^{1 *}$ T. Ünler is with Necmettin Erbakan University, Faculty of Aviation and Space Sciences, Department of Satellite Space and Sciences, Konya, Turkey

${ }^{2}$ L. Seyfi is with Selcuk University, Faculty of Engineering, Dept. of Electrical and Electronics engineering, Konya, Turkey
}

by external factors. So their outputs are more stable than VCO (Voltage Controlled Oscillator) made with transistors. There are also CMOS pulse generators designed for short distances and widely used in the car industry [6]. Digitally generated signal generator architects also have pulse generators consisting of structures such as flip-flops, multiphase pulse generators, and baluns [7]. With this structure, frequency changes can be made flexibly and digitally controllable [2], [7]

In this study, RF signals at 7 different frequencies will be produced. signals with frequencies of $137.5 \mathrm{MHz}, 400 \mathrm{MHz}$, $800 \mathrm{MHz}, 1200 \mathrm{MHz}, 1500 \mathrm{MHz}, 1800 \mathrm{MHz}$ and $1950 \mathrm{MHz}$ were produced making calculations according to the $25 \mathrm{MHz}$ reference oscillator frequency.

\section{MATERiAl AND MethoD}

In this study, ADF4350BCPZ chip which is shown in Fig. 1, was used to produce RF signal. This chip can produce RF signals which has $0.001 \mathrm{kHz}$ sensitivity between $137 \mathrm{MHz}$ and $4400 \mathrm{MHz}$ with PLL (Phase Locked Loop). Especially PLL property of this chip is distinctive among other VCOs (Voltage controlled oscillators). VCOs usually experience a frequency shift on RF signals due to temperature and other factors. Therefore, ADF4350BCPZ chip provide quite advantage at radar design. The desired frequency can be obtained by programming this chip with SPI. All configuration of this chip is created by writing data to 6 registers of 32 bit at SPI programming. Output power, modulation frequency and the other settings can be programmed via these registers. PIC18F4550, a microcontroller, was used for programming the chip. This microcontroller can work up to $48 \mathrm{MHz}$ with PLL. In the project, reference oscillator of $4 \mathrm{MHz}$ was used and microcontroller was used at $24 \mathrm{MHz}$ to write and access to registers for production of RF signal with HS (high speed) PLL. Frequency band is selected by different calculations at registers for each frequency band.

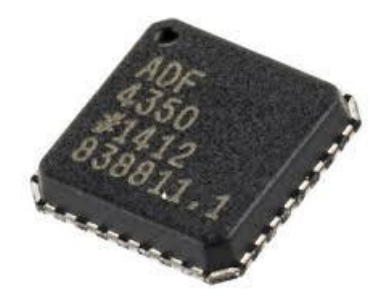

Fig. 1: ADF4350BCPZ Chip

The frequency of the signal to be produced is calculated acording to the following formulas: 
$R F_{O U T}=f_{P F D} \times(I N T+(F R A C / M O D))$

$f_{P F D}=R E F_{I N} \times[(1+D) /(R \times(1+T))]$

Here, parameters of FRAC, MOD, R, D, and INT are values which are written on registers as shown in Fig.2. $\mathrm{REF}_{\mathrm{IN}}$ is the frequency of active oscillator which is used for ADF4350BCPZ chip. FRAC and INT parameters are present in register0 as shown in Fig.2.

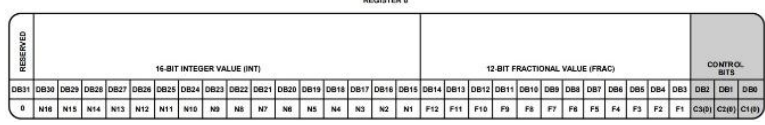

Fig. 2: Register0

12 bit-MOD value is present in register1 as shown in Fig.3

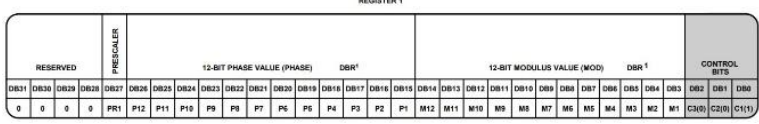

Fig. 3: Register1

10 bit - $\mathrm{R}$ counter is present in register2 as shown in Fig.4.

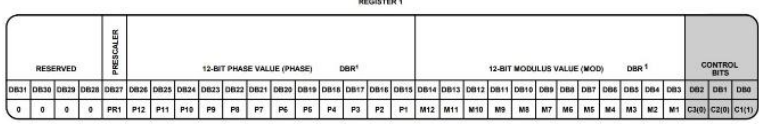

Fig. 4: Register2

Blok diagram of ADF4350BCPZ chip which is used as signal generator and register situations are given in Fig.5.

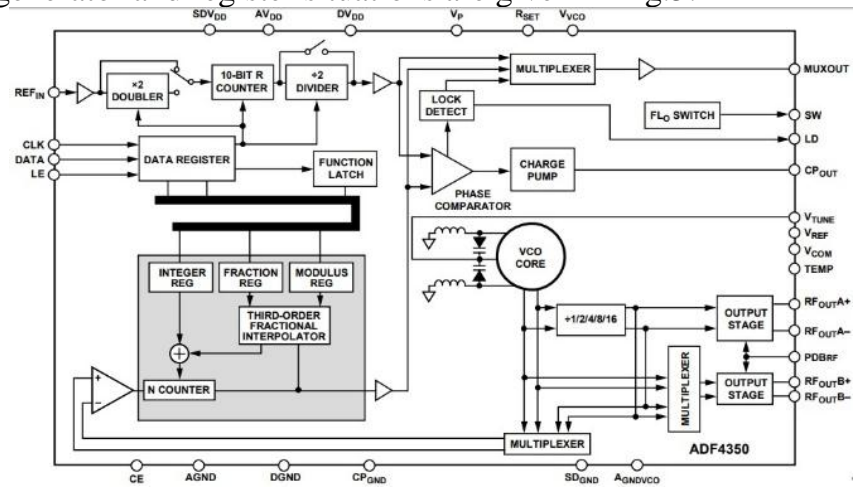

Fig. 5: ADF4350BCPZ Blok Diagram

Because project was carried out at high frequencies, appropriate SMD (Surface Mount Device) materials were chosen to be used. Connection paths of the electronic circuit were executed at a short longitude in order not to encounter an interference at especially RF part. Additionally, unnecessary turning on connection paths was mostly avoided while designing the circuit.

RF signal measurements were carried out using UNAOHM EP314 spectrum anlyzer and KC901S network analyzer which are shown in Fig.6 and Fig.7.

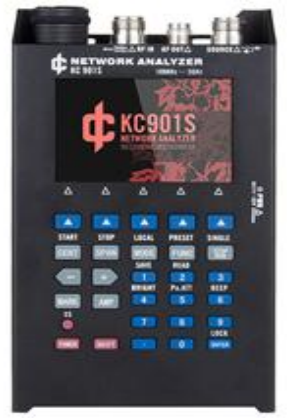

Fig. 6: KC901S Network Analyzer

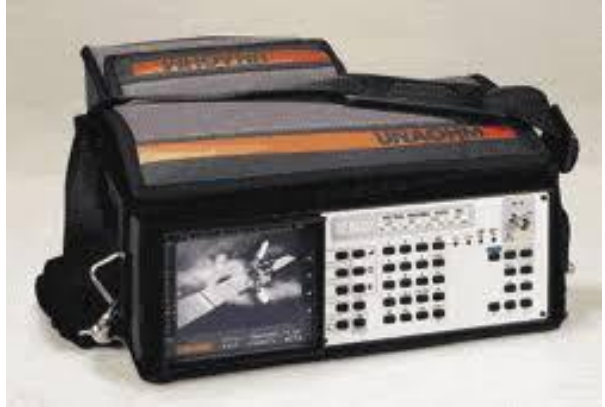

Fig. 7: UNAOHM EP314 Spektrum Analyze

Measurements of return loss, VSWR, S11, S21 can be made from the frequency of $100 \mathrm{kHz}$ to $3 \mathrm{GHz}$ by using KC901S network analyzer. Because the network analyzer contains AF and RF sources, it is possible to use it as a signal generator at desired frequency. Additionally, Smith chart calculations can visually be fulfilled via the network analyzer.

UNAOHM 314 spectrum analyzer can be used at from the frequency of $45 \mathrm{MHz}$ to $2150 \mathrm{MHz}$. In this study, UNAOHM 314 spectrum analyzer was used to compare the produced RF signal by using ADF4350 chip with the signal obtained from the network analyzer.

In addition, RIGOL1052E oscilloscope which is shown in Fig.8, was used to measure SPI and all digital inputs and outputs. This oscilloscope has a capability of measuring signals via 2 channels up to $50 \mathrm{MHz}$.

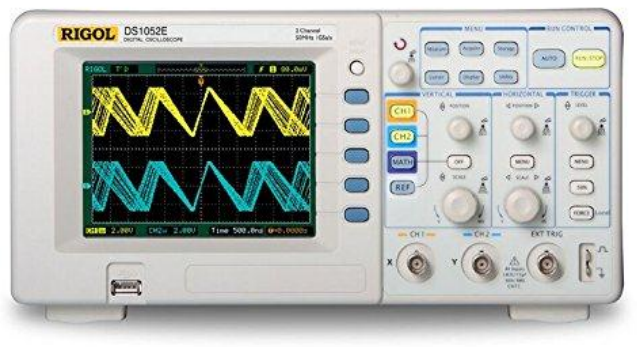

\section{A. Supply and RF Isolation}

Because LM2576-ADJ chip, which was used at supply part of the circuit, is capable of working independently from the current flowing, misworking of both microcontroller and ADF4350BCPZ chip due to variation of supply voltage was prevented. It was observed that there was not any oscillation of voltage under load at oscilloscope outputs thanks to ripple filter which was used at the output of LM2576-ADJ. Furtherly, because $74 \mathrm{HC} 244$ buffer chip has high impedance at the output, ADF4350BCPZ and microcontroller could be isolated from eachother. Output of microcontroller could be preserved due to this situation, too.

\section{B. Microcontroller}

PIC18F4550 used as a microcontroller was supplied with $4 \mathrm{MHz}$ external oscillator. Frequency was set to $24 \mathrm{MHz}$ (96 $\mathrm{MHz} / 4)$ via PLL property of the chip. SPI signals were transferred over transmission line and 74HC244 chip to ADF4350BCPZ chip after it was successfully produced. SPI signals were observed at microcontroller output, 74HC244 output and ADF4350BCPZ input with oscilloscope. It was observed that the signals were transferred to the 3 points without 
any deformation.

\section{Production AND MEASUREMENT OF RADAR Signals}

Firstly, circuit design was performed by using Proteus. Then, the circuit was put into practice. Supply voltage of $3.3 \mathrm{~V}$ was created from input voltage of $12 \mathrm{~V}$ via LM2576-ADJ, which is a step-down switching voltage regulator chip. Both PIC18F4550 and $74 \mathrm{HC} 244$ were supplied with this voltage. As shown in Fig.9, output frequency of active oscillator which was used for ADF4350BCPZ chip was measured as $25 \mathrm{MHz}$.

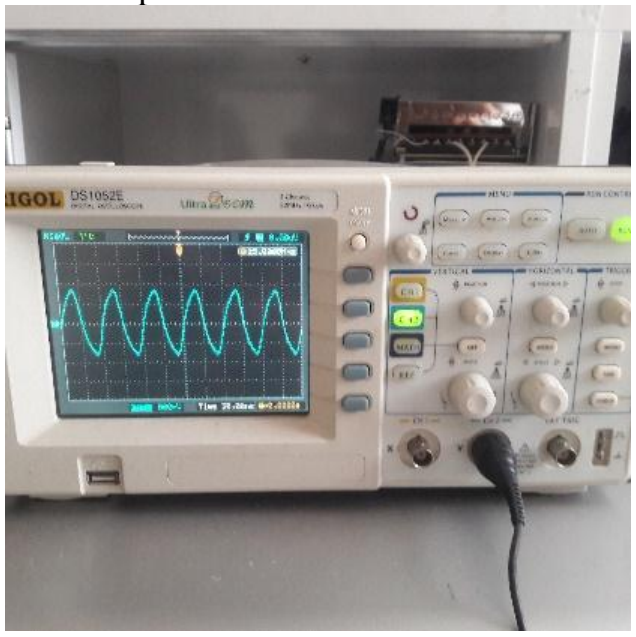

Fig. 9: Observation of 25MHz reference oscillator frequency for ADF4350

74HC244 chip was used to isolate ADF4350BCPZ chip from $18 \mathrm{f} 4550$ microcontroller. Therefore, it was possible to construct a stabilized system which was not affected from each other. At this level, SPI signal was produced at SPI module of the microcontroller with 3 pins and transmitted to ADF4350BCPZ chip. SPI signals were measured with oscilloscope.

32 bits operations were executed on the microcontroller by shifting operation of 8 bits four times. Additionally, signal output of ADF4350BCPZ was faded by using CE (chip enable) input. Therefore, duty cycle of radar signals can be organized in software.

Bottom appearance of the printed circuit board (PCB) which was manufactured and assembled is shown in Fig. 10.

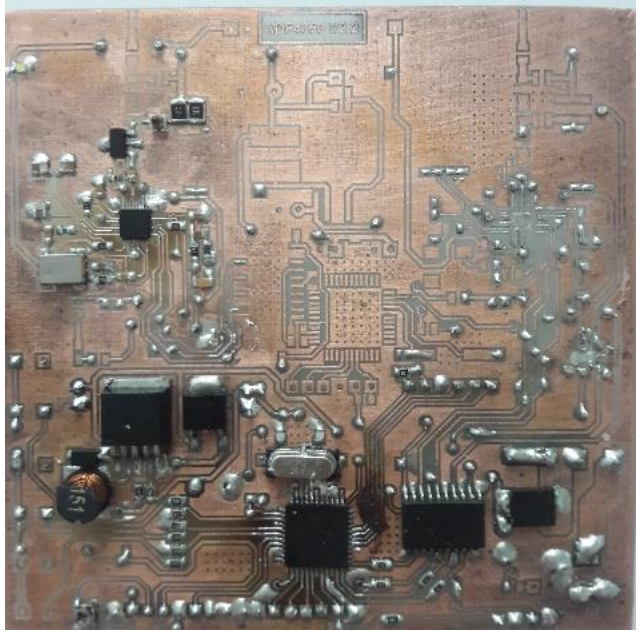

Fig. 10: Bottom appearance of the printed circuit board
Top appearance of the PCB is shown in Fig.11.

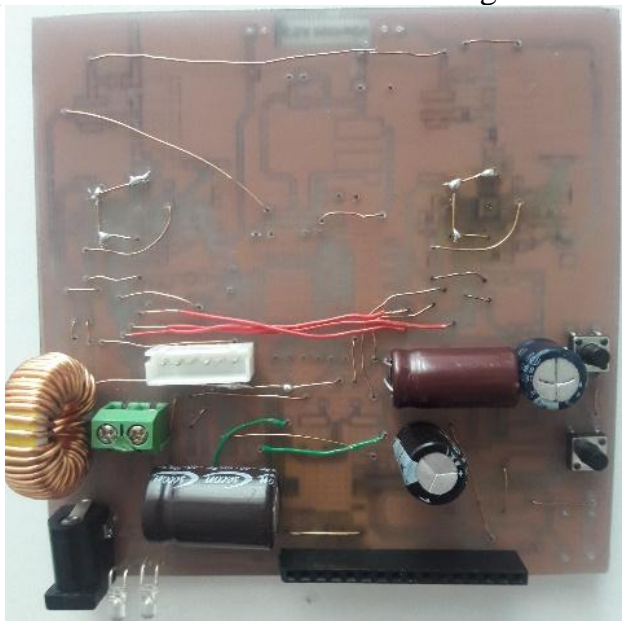

Fig. 11: Top appearance of the printed circuit board

The produced RF signal at $137.5 \mathrm{MHz}$ is shown in Fig. 12.

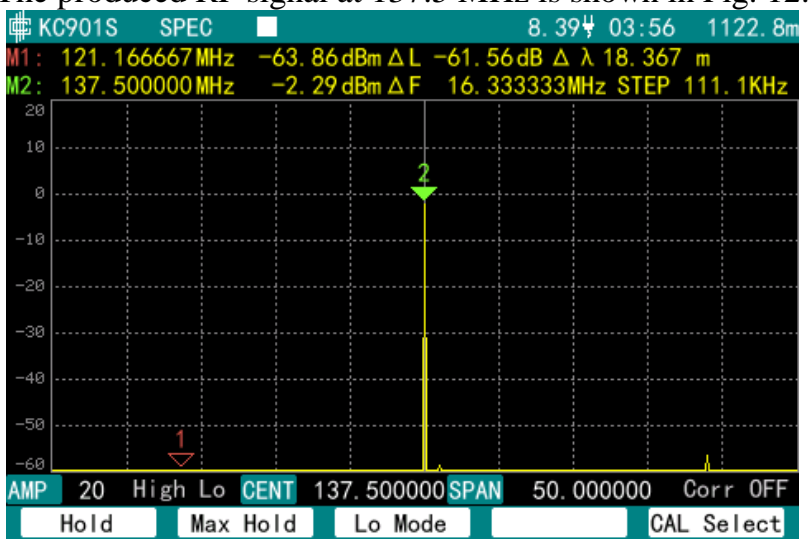

Fig. 12: The produced RF signal at $137.5 \mathrm{MHz}$

\section{CONCLUSION}

As a result of this study, radar signals were obtained from manufactured PCB at predetermined 7 frequencies. Because frequency setting of the produced signal can be fulfilled in software, signals at different frequency can be produced sequentially. Since the signals were produced with PLL, they had stabilized frequencies. Additionally, because communication of the chip [hangisi] was provided via SPI protocol, variation of frequency and other properties of the signal can be so rapidly carried out that this takes advantage at applications of radar such as MIMO in which radar frequency is required to be rapidly changed. SPI protocol theoretically provides $100 \mathrm{Kbt} / \mathrm{s}, 400 \mathrm{~Kb} / \mathrm{s}, 1 \mathrm{Mb} / \mathrm{s}$, and $3.2 \mathrm{Mb} / \mathrm{s}$ in Low speed, full speed, fast, and high speed modes, respectively [1]. Because the signals having properties of last register data was observed at the output of ADF4350BCPZ chip when there was not SPI communication and even data, CE pin of the chip was preferred to make RF signal active and passive at the output. Signal output of the radar can be off thanks to using CE pin at the time when radar is used to receive echo signal without interference of the transmitted signal. 


\section{REFERENCES}

[1] T. Norimatsu, R. Fujiwara, M. Kokubo, "A UWB-IR Transmitter with Digitally Controlled Pulse Generator" IEEE Journal of Solid-State Circuits, Vol. 42, No. 6, JUNE 2007.

[2] P. Hemnani, A.K. Rajarajanb, G. joshic, S.V.G. Ravindranathd "FPGA based RF pulse generator for NQR/NMR spectrometer", Procedia Computer Science, 93 (2016), pp. 161 - 168, 2016.

[3] A.Yıldırım, E. Yaldız, "Pulse Generator Design for UWB Applications" in Proc. Electrical, Electronics, Computer and Biomedical Egnineering Conf (Eleco 2014), 27 - 29 November 2014.

[4] F. Zito, D. Pepe, D. Zito, "UWB CMOS Monocycle Pulse Generator" IEEE Transactions on Circuits and Systems-I: Regular Papers, Vol. 57, No. 10, pp. 2654 - 2664, October 2010. https://doi.org/10.1109/TCSI.2010.2047751

[5] D. Zito, D. Pepe, M. Mincica, F. Zito, A. Tognetti, A. Lanatà, and D. De Rossi, "SoC CMOS UWB Pulse Radar Sensor for Contactless Respiratory Rate Monitoring" IEEE Transactions on biomedical Circuits and Systems, Vol. 5, No. 6, pp. 503 - 510, December 2011. https://doi.org/10.1109/TBCAS.2011.2176937

[6] A. Oncu, W. Badalawa, M. Fujishima, "22-29 GHz Ultra-Wideband CMOS Pulse Generator for Short-Range Radar Applications" IEEE Journal of Solid-State Circuits, Vol. 42, No. 7, pp. 1464 - 1471, July 2007.

https://doi.org/10.1109/JSSC.2007.899099

[7] L. Smaïni, C. Tinella, D. Hélal, C. Stoecklin, L. Chabert, C. Devaucelle, R. Cattenoz, N. Rinaldi, and D. Belot, "Single-Chip CMOS Pulse Generator for UWB Systems", IEEE Journal of Solid-State Circuits, Vol. 41, No. 7, pp. 1551 - 1561, July 2006.

https://doi.org/10.1109/JSSC.2006.873896 